\title{
1 Association of the gut microbiota with colorectal cancer in a South
}

\section{Asian cohort of patients}

3 AGP Sahankumari ${ }^{1}$, Bawantha D. Gamage ${ }^{1 *}$, Gathsaurie Neelika Malavige ${ }^{2}$.

$4{ }^{1}$ Department of Surgery, Faculty of Medical Sciences, University of Sri Jayewardanepura,

$5 \quad$ Nugegoda, Sri Lanka

$6{ }^{2}$ Department of Microbiology, Faculty of Medical Sciences, University of Sri Jayewardanepura,

$7 \quad$ Nugegoda, Sri Lanka

8

9 Corresponding author*:

10

Dr. Bawantha Gamage, MBBS,MRCS,MS,FAMASI,FCSSL

11

Department of Surgery, Faculty of Medical Sciences,

12

University of Sri Jayewardanepura,

13 Nugegoda, Sri Lanka

14

P.O. Box 10250

15

Email:bawantha@sjp.ac.lk

17 Keywords: Colorectal cancer, gut microbiome, diabetes, Bacteroides fragilis, Akkermansia 


\section{Abstract}

Background: As the gut microbiome is thought to play a role in the pathogenesis of colorectal carcinoma (CRC) and affected by the diet and the genetic composition, we sought to investigate the patterns of gut microbiota that associate with CRC in a South Asian cohort of patients with CRC.

Methodology: The relative abundance of 45 types of gut microbial species were determined in faecal samples of CRC patients $(n=24), D M(n=20)$ and healthy age matched controls $(n=44)$, using a PCR array. Data was analyzed using the specific software for analysis of bacterial DNA quantification.

Results: The species Bacteroides fragilis (23.9-fold), Bacteroides thetaiotaomicron (8-fold) and Akkermansia muciniphila (5.9 fold) were several-fold over expressed in patients with CRC compared to healthy individuals, whereas bacterial species of the Phylum Proteobactria were under expressed. There was no difference in the abundance of these 3 species of bacteria with tumour stage or gender and age of patients. Aeromonas species, Enterococcus faecium and Shigella dysenteriae (Proteobacteria) were over 100-fold over abundant in those with DM compared to healthy individuals. Although $70.83 \%$ of those with $\mathrm{CRC}$ also had diabetes, the relative abundance of microbiota in CRC patients were different to those who had diabetes and no CRC.

Conclusions: Patients with CRC and DM harbor a markedly different gut microbiota patterns compared to their healthy counterparts. Similar patterns of gut microbial dysbiosis that associate with CRC and DM appear be seen in South Asian populations, compared to Western countries, despite differences in the diet and ethnicity. 


\section{Background}

42 Colorectal cancer (CRC) is the third commonest cause of cancer worldwide and is the fourth

43 commonest cancer leading to death [1]. It has been predicted that the deaths due to colonic cancer

44 and rectal cancer will increase by $60 \%$ and $71.5 \%$, respectively until year 2035 due to the increase

45 in the aging population [1]. The increase in the incidence of CRC is predicted to rise substantially

46 more in developing countries vs developed countries due to these changes in population

47 demographics[2]. As this increase in the incidence of CRC in developing countries is likely to

48 result in a huge burden to their economies, there is an urgent need to implement programs that

49 reduce its occurrence and adopt novel diagnostic and treatment methods of CRC.

51 Diet and lifestyle are major risk factors for development of CRC along with genetic susceptibility,

52 the presence of metabolic diseases such as diabetes and obesity and inflammatory bowel disease

53 [3-6]. Metabolic diseases and CRC are associated with microbial dysbiosis, which is characterized

54 reduced diversity of the gut microbiome with an overabundance of the genera Proteobacteria and

55 Firmicutes [7]. Several types of microbiota have been shown to associate with CRC such as pks-

56 positive E.coli, enterotoxigenic Bacteriodes fragilis, Fusobacterium nucleatum and Streptococcus

57 gallolyticus [8-12]. While some of these microbes were overabundant in the gut microbiome of

58 patients with $\mathrm{CRC}$, some have been detected specifically in tumor tissue and also in distance

59 metastasis, suggesting that they may play a role in the pathogenesis of this cancer [12]. They are

60 thought predispose to the development of $\mathrm{CRC}$ by inducing epigenetic changes and thereby

61 affecting gene transcription, inducing DNA damage and reactive oxygen species and by inducing

62 procarcinogenic cytokines [12]. 
63 Of the factors that affect the diversity of the gut microbiome, the diet plays a central role. Although

64 the relative abundance of gut microbiota depends on an individual's genetic composition (12\%),

65 the influence of the diet is much greater (57\%)[13]. Individuals who predominantly consume a

66 Mediterranean diet, rich in grains, legumes, nuts, vegetable and fruits were found to have a gut

67 microbiome which reduced the risk of metabolic diseases, inflammatory bowel disease and colonic

68 cancer compared to those who consume a typical Western diet [14]. South Asian individuals have

69 a very different diet than those of Western and the South East Asian populations, due to differences

70 in religious and cultural practices. Their diets are typically rich in grains, pulses, vegetables and

71 fruits with a low intake of red meat. These vast differences in the diet are likely to influence the

72 microbial composition and thus protect or predispose to the development of CRC. In fact, is has

73 been shown that the gut microbiome is significantly different in individuals of different ethnicity,

74 living in the same geographical area [15]. Since dietary factors are likely to directly contribute to

75 the microbial composition and thus to the risk of developing $\mathrm{CRC}$, change in the dietary patterns

76 can be an important strategy in the prevention and treatment of $\mathrm{CRC}[16]$. Therefore, in order to

77 implement such preventive and therapeutic strategies, it would be important to initially

78 characterize the gut microbial patterns in South Asian individuals with CRC living in those 79 countries.

81 In this study, we have determined the relative abundance of 45 species of gut microbiota on

82 patients with CRC, aged matched healthy individuals and also in patients with metabolic diseases

83 such as diabetes. We found that that pattern of the gut microbiota was significantly different in

84 those with CRC and diabetes compared to healthy individuals. 


\section{Methods}

86

87

88

89

90

91

92

93

94

95

96

97

98

99

100

101

102

103

104

105

106

107

\section{Patients}

We recruited 24 patients with CRC who underwent colonoscopy at Colombo South Teaching Hospital, Sri Lanka between January 2017 and April 2018, following informed written consent. Stool samples were obtained from these patients two weeks after colonoscopy. All clinical details regarding altered bowel habits, abdominal pain, loss of weight, appetite along with laboratory and radiological investigations such full blood count, ultrasound scanning of the abdomen and CT scans were recorded and CRC grading was carried out according to TNM staging classification $[17]$

\section{Recruitment of healthy controls and patients with diabetes mellitus}

In order to compare the changes that associate with $\mathrm{CRC}$, we recruited healthy individuals $(\mathrm{n}=44)$ who underwent colonoscopy and were found not to have any bowel pathology and who were nonobese, (BMI $<23.9$ ), waist circumference $<80 \mathrm{~cm}$ for females and $<90 \mathrm{~cm}$ for males and who did not have diabetes or hyperlipidemia. Again, the stool samples were obtained from these patients two weeks after colonoscopy.

In addition to the above controls, as most of the patients with $\mathrm{CRC}$ also had diabetes mellitus (DM), in order to differentiate the changes in the patterns of gut microbiota with those that are specific to CRC, we also recruited patients with DM $(n=20)$ who underwent colonoscopy and were found not to have any CRC or gut pathology, between January 2017 to April 2018. As in patients with CRC, stool samples were obtained from these individuals, two weeks following colonoscopy. Patients who had adenomas, who received antibiotic therapy for more than 1 week 
108 prior to stool sample collection, who received chemotherapy and/or radiation or with a history of

109 CRC and inflammatory bowel disease were excluded.

\section{Ethics Statement}

112 All subjects provided informed written consent prior to participating in the study. Ethical approval 113 was granted by Ethical Review Committee, Faculty of Medical Sciences, University of Sri 114 Jayewardenepura (Application No: 35/16).

\section{Sample collection and DNA Extraction from stools}

117 The stool samples were collected two weeks after the patients underwent colonoscopy giving time

118 for the gut microbiota to re-establish itself. The stool samples were transported to the laboratory 119 within 24 hours after collection. DNA was extracted using QIAamp DNA Stool Mini Kit (QS, 120 Hilden, Germany) according to the manufacturer's instructions and the extracted DNA stored at $12120^{\circ} \mathrm{C}$ prior to quantification of the stool microbiota.

\section{Quantification of the gut microbiota}

125 The Microbial DNA qPCR Array Intestinal Infection 2 kit (Qiagen, Hilden, Germany)

126 (Supplementary table 1) was used to amplify species specific 16S rRNA genes in order to identify 127 and to quantify the relative abundance of 45 types of gut microbiota according to the 128 manufacturer's instructions (Qiagen, Hilden Germany) (Supplementary table 1). Briefly, 5000ng 129 (0.005 mg) extracted bacterial DNA from each stool specimen was used in the microbial qPCR 
master mix and target-specific fluorescent probes. The reaction was performed in an Applied Biosystems 7500, 96-well plate detection system. qPCR cycling condition first step comprise of

132 one cycle of initial PCR activation step for 10 minutes at $95^{\circ} \mathrm{C}$, following 40 cycles of denaturation

133 for $15 \mathrm{sec}$ at $95^{\circ} \mathrm{C}$ and annealing and extension for 2 minutes at $60^{\circ} \mathrm{C}$ as the second cycling reaction

134 step. The threshold cycle value $(\mathrm{Ct})$ for each reaction was determined by manually setting the

135 threshold limit. The relative abundance of different bacterial species was determined by comparing

136 the $\mathrm{Ct}$ value of each bacterial species with the $\mathrm{Ct}$ values of same bacterial species generated from

137 the healthy controls using the Baid 1407 intestinal infections microbial profiling data analysis 138 software (Qiagen, Hilden Germany).

\section{Statistical analysis}

141 Statistical analysis was performed using Graph PRISM version 7. Differences in the relative

142 abundance of different bacterial species in patients with CRC, patients with diabetes and healthy

143 individuals were compared using the Mann-Whitney U test (two tailed), 


\section{Results}

\section{Characteristics of patients with CRC}

155 Of the 24 patients with CRC, $15(62.5 \%)$ were males and $9(37.5 \%)$ were females. The median 156 age of those with CRC was 59 years (IQR 53.25 to 64 years), in those with DM was 65 (IQR 59.5 157 to 68 years) and in healthy individuals was 53 (IQR 47 to 65 years). In patients with CRC, 4/24 $158(16.67 \%)$ of the tumors were present in the descending colon, $4 / 24(16.67 \%)$ in the sigmoid colon, $15911 / 24(45.83 \%)$ in the rectum, $2 / 24(8.33 \%)$ in recto -sigmoid junction and $1 / 24(4.17 \%)$ in hepatic 160 flexure, $1 / 24(4.17 \%)$ in anal verge and 1/24 (4.17\%) in transverse colon. 17/24 (70.83\%) of those 161 with CRC had DM.

162

$1632 / 24$ of CRC patients were in stage 0 and $\mathrm{i}$ based on the TNM classification at the time of diagnosis, 1647 were in stage ii, $8 \mathrm{~s}$ in stage iii and 7 patients were in stage iv. None of the patients with CRC, 165 DM or the healthy individuals had a previous history of inflammatory bowel disease or CRC. The 166 clinical details of all patients with CRC is shown in Table 1. 


\begin{tabular}{|c|c|c|c|c|c|}
\hline Patient ID & Gender & Age & $\begin{array}{c}\text { Tumour } \\
\text { stage }\end{array}$ & Tumour location & $\begin{array}{l}\text { Diabetes } \\
\text { mellitus }\end{array}$ \\
\hline 1 & $\mathrm{M}$ & 74 & 0 & rectum & $\mathrm{DM}+$ \\
\hline 2 & $\mathrm{M}$ & 62 & iii & sigmoid colon & $\mathrm{DM}+$ \\
\hline 3 & $\mathrm{M}$ & 68 & $\mathrm{i}$ & hepatic flexture & $\mathrm{DM}+$ \\
\hline 4 & $\mathrm{M}$ & 67 & ii & rectum & $\mathrm{DM}+$ \\
\hline 5 & $\bar{F}$ & 54 & ii & rectum & $\mathrm{DM}+$ \\
\hline 6 & $\bar{F}$ & 43 & ii & rectum & DM0 \\
\hline 7 & $\mathrm{M}$ & 54 & ii & Rectum & DM0 \\
\hline 8 & $\mathrm{M}$ & 59 & ii & $\begin{array}{c}\text { Recto - sigmoid } \\
\text { junction }\end{array}$ & $\mathrm{DM}+$ \\
\hline 9 & $\mathrm{M}$ & 91 & ii & desending colon & $\mathrm{DM}+$ \\
\hline 10 & $\mathrm{~F}$ & 43 & ii & rectum & DM0 \\
\hline 11 & $\mathrm{M}$ & 48 & iv & $\begin{array}{l}\text { Lower and mid } \\
\text { rectal CA }\end{array}$ & DM0 \\
\hline 12 & $\mathrm{M}$ & 64 & iii & lower rectum & $\mathrm{DM}+$ \\
\hline 13 & $\mathrm{~F}$ & 64 & iii & anal verge & $\mathrm{DM}+$ \\
\hline
\end{tabular}




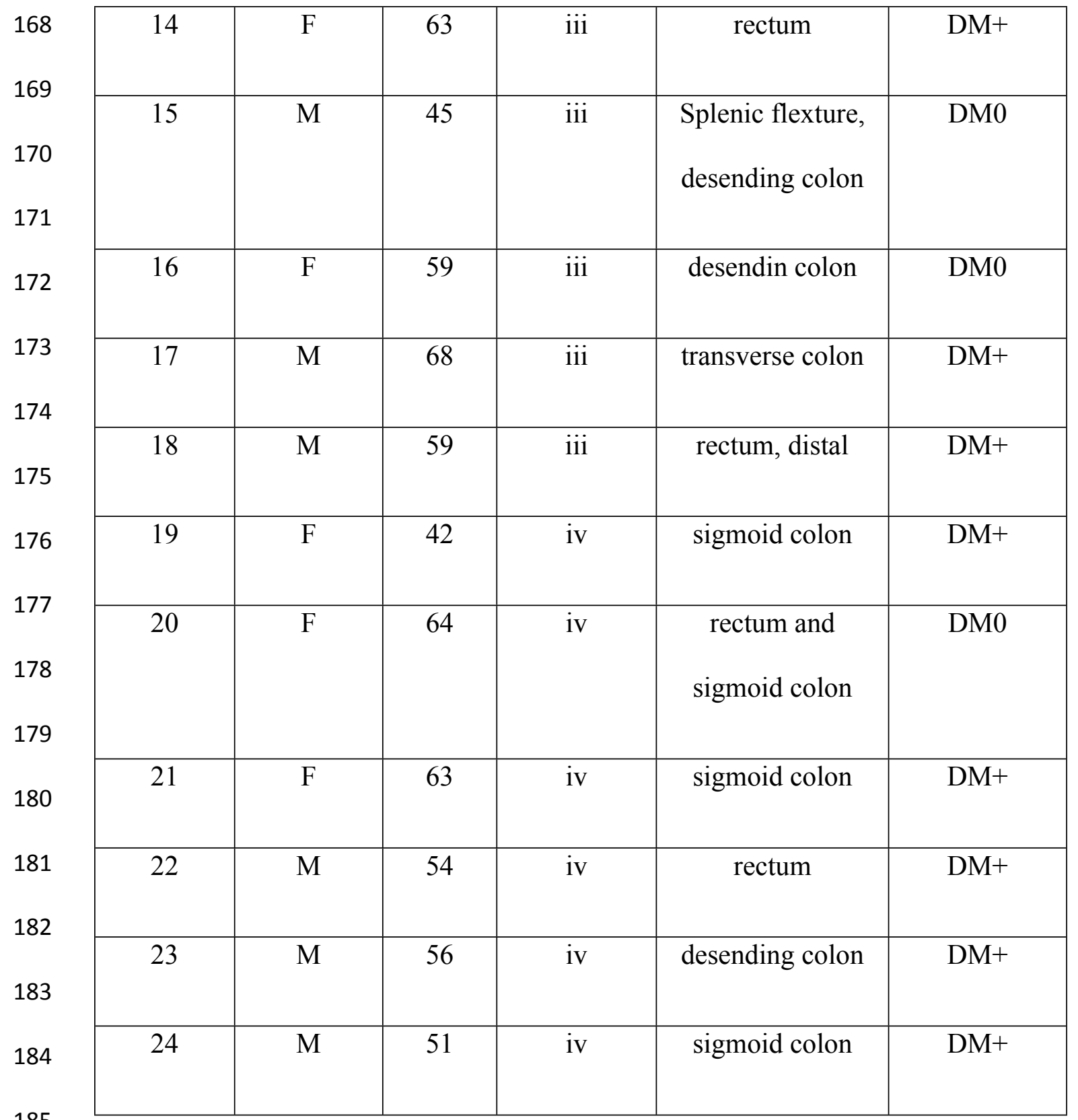

186

187 


\section{Patterns of gut microbiota in CRC compared to healthy individuals}

192 As the relative abundance of gut microbiota has shown to be markedly different in those with CRC compared to healthy individuals ${ }^{7}$, we proceeded to determine the patterns of gut microbiota in our

194 cohort of patients, in comparison to healthy individuals.

We observed marked differences in the gut microbiota patterns in patients with CRC when

thetaiotaomicron, Akkermansia muciniphila, Aeromonas spp. (Aeromonas enteropelogenes,

1). Most notably Bacteroides fragilis was 23.9-fold over expressed in those with CRC compared

Figure 1: Gut microbial patterns of patients with CRC compared to healthy individuals. The relative abundance of 45 gut microbiota species in patients with CRC $(n=24)$ and healthy individuals $(\mathrm{n}=44)$ were investigated using a PCR array amplifying the $16 \mathrm{~S}$ rRNA genes in stool samples. The relative abundance of each microbial species relative to the abundance in healthy volunteers is shown.

\section{Changes in the patterns of gut microbiota in patients with diabetes mellitus when compared to healthy individuals}

The relative abundance of the gut microbiota has shown to be different in those with metabolic diseases such as DM $[7,18]$. As 17/24 (70.83\%) patients with CRC also had DM, we proceed to 
determine if these changes in the gut microbiota observed in those with CRC were associated with CRC or with DM. In order to determine the changes associated with DM, we compared the patterns of gut microbiota in patients with DM (n=20) with the group of healthy volunteers $(n=44)$. Again. we observed marked differences in the gut microbiota pattern in patients with DM compared to healthy individuals (Fig 2).

Figure 2: Gut microbial patterns of patients with DM compared to healthy individuals. The relative abundance of 45 gut microbiota species in patients with DM $(n=20)$ and healthy individuals $(\mathrm{n}=44)$ were investigated using a PCR array amplifying the $16 \mathrm{~S}$ rRNA genes in stool samples. The relative abundance of each microbial species relative to the abundance in healthy volunteers is shown.

Aeromonas spp. (Aeromonas enteropelogenes, Aeromonas hydrophila, Aeromonas punctate, Aeromonas media) were the predominant microbes, in patients with DM, which were seen at 226.64-fold times higher than healthy individuals. The abundance of Enterococcus faecium, Bacteroides thetaiotaomicron, Streptococcus agalactiae. Shigella dysenteriae, Enterococcus faecalis, Bacteroides fragilis and Plesiomonas shigelloides were also several fold higher in the stool samples of DM patients compared to healthy individuals (Table 2) followed by E. faecium, S. dysenteriae and Streptococcus agalactiae. In contrast, to what we observed in patients with CRC, all the 45 bacterial species investigated several folds more abundant in patients with DM compared to healthy individuals. 


\section{Comparison of the gut microbial patterns of CRC patients compared to those with DM}

As we observed marked differences the relative abundance of the gut microbiota between those with CRC and healthy individuals and those with DM and healthy individuals, and since 17/24 (70.83\%) of the individuals with CRC had DM, we proceeded to analyze the gut microbial patterns specific to CRC by comparing the microbial patterns of those with CRC $(n=24)$ with those with $\operatorname{DM}(n=20)$.

Although all 45 bacterial species were overabundant in patients with DM compared to those with CRC, of whom 70.8\% had DM, Bacteroides fragilis, thetaiotaomicron and Akkermansia muciniphila were least expressed (Figure 3). As, these 3 species of bacteria appear to be associated with CRC, we evaluated the expression of these 3 species related to tumour grade and site of the tumor. There was no difference in the abundance of these 3 species of bacteria with the stage 0 to ii $(n=9)$, iii $(n=9)$ and iv ( $=6)$, when analysed using the Kruskal-Wallis test. There was also no difference in their abundance based on gender or age.

Figure 3: Gut microbial patterns of patients with CRC compared to patients with DM. The relative abundance of 45 gut microbiota species in patients with $\mathrm{CRC}(\mathrm{n}=24)$ and patients with diabetes mellitus $(n=20)$ were investigated using a PCR array amplifying the 16S rRNA genes in stool samples. The relative abundance of each microbial species relative to the abundance in healthy volunteers is shown. 


\section{Discussion}

In this study we investigated the differences in the gut microbial patterns in a South Asian cohort of individuals with $\mathrm{CRC}$ and found that the gut microbial patterns were vastly different between those with CRC and healthy individuals. As a large proportion (70.83\%) of those with CRC also had diabetes, in order to determine if the changes in the relative abundance of different bacteria were due to the presence of diabetes, we compared the gut microbial patterns in patients with DM with age matched healthy individuals. Again, we found that the gut microbial patterns were indeed markedly different in those with DM compared to healthy individuals. Further comparison of the microbial patients with DM with those with CRC showed that three bacterial species were more likely to be associated with CRC. These are Bacteroides fragilis, Bacteroides thetaiotaomicron and Akkermansia muciniphila. However, the relative abundance of these three bacterial species did not differ based on the tumour stage, age or the gender of patients.

Many previous studies have shown the association between CRC and Bacteroides fragilis [19]. The toxin produced by Enterotoxigenic Bacteroides fragilis has shown to change gene transcription in the colons of mice models by inducing epigenetic changes, which subsequently result in development of tumors [20]. The toxin producing strains of Bacteroides fragilis has shown to be more prevalent in patients with CRC [10]. Although we did not specifically assess toxin production by Bacteroides fragilis in this study, this bacterium was found to be 23.9 times overabundant in those with CRC compared to healthy individuals. Although we found that Bacteroides vulgatus was also two-fold over abundant in those with CRC compared to healthy 
individuals, other studies have shown that Bacteroides vulgatus was more abundant in healthy individuals [21]. In addition, we found that Bacteroides thetaiotaomicron was 8-fold more over abundant in those with CRC when compared to those with CRC, which has not been reported before.

Ethnic differences have shown to play a significant role in the outcome of $\mathrm{CRC}$, which has been attributed to possible differences in the gut microbiome [22]. For instance, while Bacteroides species, Fusobacterium nucleatum and Enterobacter species were more abundant in AfricanAmerican patients with CRC, Akkermansia muciniphila and Bifidobacterium species were more abundant in Caucasians [22]. As our PCR array did not have Fusobacterium nucleatum and Bifidobacterium species included, we could not assess their relative abundance. However, Akkermansia muciniphila was 5.9-fold over abundant in those with CRC, compared to healthy individuals. Therefore, it would be important to carry out $16 \mathrm{~S}$ sequencing of the whole gut microbiome to derive better data to find out the microbiota that associate with CRC in the Sri Lankan population.

As $70.8 \%$ of the CRC patients also had DM, in order to identify the gut microbiota patterns related to DM, we assessed the patterns between healthy individuals and those with DM. We found that all the 45 types of gut microbiota assessed in this study, were several folds over expressed in those with DM compared to healthy individuals. Specifically, the Aeromonas species, Enterococcus faecium and Shigella dysenteriae were over 100-fold over abundant in those with DM compared to healthy individuals. Therefore, bacteria of the Phylum Proteobacteria do appear to be 100-fold 
more abundant in South Asian patients with DM, similar to the observations in Western countries, despite differences in their diets [23]. This gut microbial dysbiosis that occurs due to the overgrowth of bacteria of the Phylum Proteobacteria has shown to associate with low grade endotoxaemia [24]. The presence of low levels of bacterial lipopolysaccharide (LPS) has shown to associate with DM and other metabolic diseases such as non-alcoholic steatohepatitis, which are rapidly increasing in all South Asian countries [25-27]. Therefore, similar patterns of gut microbial dysbiosis that associate with CRC and DM appear be seen in South Asian populations as in other countries, despite differences in the diet and ethnicity.

In summary, we studied the gut microbial patterns in a South Asian cohort of patients with CRC and found that Bacteroides fragilis, Bacteroides thetaiotaomicron and Akkermansia muciniphila were several folds over abundant in those with CRC when compared to healthy individuals. However, as this study was limited to studying only 45 genera and species of microbiota, it would be important to study the whole gut microbiome by carrying out $16 \mathrm{~S}$ sequencing to identify other possible microbes that associate with CRC. Early identification of such gut microbial dysbiosis could lead to prevention and treatment strategies in populations by possible nutrition interventions. 


\section{References:}

1. Araghi M, Soerjomataram I, Jenkins M, Brierley J, Morris E, Bray F, et al. Global trends in colorectal cancer mortality: projections to the year 2035. Int J Cancer. 2018. doi: 10.1002/ijc.32055. PubMed PMID: 30536395.

2. Tsoi KKF, Hirai HW, Chan FCH, Griffiths S, Sung JJY. Predicted Increases in Incidence of Colorectal Cancer in Developed and Developing Regions, in Association With Ageing Populations. Clin Gastroenterol Hepatol. 2017;15(6):892-900 e4. doi: 10.1016/j.cgh.2016.09.155. PubMed PMID: 27720911.

3. Cornish AJ, Tomlinson IPM, Houlston RS. Mendelian randomisation: A powerful and inexpensive method for identifying and excluding non-genetic risk factors for colorectal cancer. Mol Aspects Med. 2019. doi: 10.1016/j.mam.2019.01.002. PubMed PMID: 30710596.

4. Banjari I, Kozic S. Dietary intake of vitamin B12 in relation to diet and lifestyle characteristics in a population at high risk for colorectal cancer. Cent Eur J Public Health. 2018;26(4):253-9. doi: 10.21101/cejph.a4585. PubMed PMID: 30660133.

5. Ballotari P, Vicentini M, Manicardi V, Gallo M, Chiatamone Ranieri S, Greci M, et al. Diabetes and risk of cancer incidence: results from a population-based cohort study in northern Italy. BMC Cancer. 2017;17(1):703. doi: 10.1186/s12885-017-3696-4. PubMed PMID: 29070034; PubMed Central PMCID: PMCPMC5657107.

6. Beaugerie L, Svrcek M, Seksik P, Bouvier AM, Simon T, Allez M, et al. Risk of colorectal high-grade dysplasia and cancer in a prospective observational cohort of patients with inflammatory bowel disease. Gastroenterology. 2013;145(1):166-75 e8. doi: 10.1053/j.gastro.2013.03.044. PubMed PMID: 23541909. 
7. Kho ZY, Lal SK. The Human Gut Microbiome - A Potential Controller of Wellness and

Disease. Front Microbiol. 2018;9:1835. doi: 10.3389/fmicb.2018.01835. PubMed PMID: 30154767; PubMed Central PMCID: PMCPMC6102370.

8. Shimpoh T, Hirata Y, Ihara S, Suzuki N, Kinoshita H, Hayakawa Y, et al. Prevalence of pks-positive Escherichia coli in Japanese patients with or without colorectal cancer. Gut Pathog. 2017;9:35. doi: 10.1186/s13099-017-0185-x. PubMed PMID: 28616082; PubMed Central PMCID: PMCPMC5468999.

9. Bonnet M, Buc E, Sauvanet P, Darcha C, Dubois D, Pereira B, et al. Colonization of the human gut by E. coli and colorectal cancer risk. Clinical cancer research : an official journal of the American Association for Cancer Research. 2014;20(4):859-67. doi: 10.1158/1078-0432.CCR13-1343. PubMed PMID: 24334760.

10. Boleij A, Hechenbleikner EM, Goodwin AC, Badani R, Stein EM, Lazarev MG, et al. The Bacteroides fragilis toxin gene is prevalent in the colon mucosa of colorectal cancer patients. Clin Infect Dis. 2015;60(2):208-15. doi: 10.1093/cid/ciu787. PubMed PMID: 25305284; PubMed Central PMCID: PMCPMC4351371.

11. Flanagan L, Schmid J, Ebert M, Soucek P, Kunicka T, Liska V, et al. Fusobacterium nucleatum associates with stages of colorectal neoplasia development, colorectal cancer and disease outcome. European journal of clinical microbiology \& infectious diseases : official publication of the European Society of Clinical Microbiology. 2014;33(8):1381-90. doi: 10.1007/s10096-014-2081-3. PubMed PMID: 24599709.

12. Allen J, Sears CL. Impact of the gut microbiome on the genome and epigenome of colon epithelial cells: contributions to colorectal cancer development. Genome Med. 2019;11(1):11. doi: 10.1186/s13073-019-0621-2. PubMed PMID: 30803449. 
13. Moreno-Indias I, Cardona F, Tinahones FJ, Queipo-Ortuno MI. Impact of the gut microbiota on the development of obesity and type 2 diabetes mellitus. Front Microbiol. 2014;5:190. doi: 10.3389/fmicb.2014.00190. PubMed PMID: 24808896; PubMed Central PMCID: PMCPMC4010744.

14. De Filippis F, Pellegrini N, Vannini L, Jeffery IB, La Storia A, Laghi L, et al. High-level adherence to a Mediterranean diet beneficially impacts the gut microbiota and associated metabolome. Gut. 2016;65(11):1812-21. doi: 10.1136/gutjnl-2015-309957. PubMed PMID: 26416813.

15. Deschasaux M, Bouter KE, Prodan A, Levin E, Groen AK, Herrema H, et al. Depicting the composition of gut microbiota in a population with varied ethnic origins but shared geography. Nature medicine. 2018;24(10):1526-31. doi: 10.1038/s41591-018-0160-1. PubMed PMID: 30150717.

16. Louis P, Hold GL, Flint HJ. The gut microbiota, bacterial metabolites and colorectal cancer. Nature reviews. 2014;12(10):661-72. doi: 10.1038/nrmicro3344. PubMed PMID: 25198138.

17. Compton C, Fenoglio-Preiser CM, Pettigrew N, Fielding LP. American Joint Committee on Cancer Prognostic Factors Consensus Conference: Colorectal Working Group. Cancer. 2000;88(7):1739-57. PubMed PMID: 10738234.

18. Dugas LR, Lie L, Plange-Rhule J, Bedu-Addo K, Bovet P, Lambert EV, et al. Gut microbiota, short chain fatty acids, and obesity across the epidemiologic transition: the METSMicrobiome study protocol. BMC public health. 2018;18(1):978. doi: 10.1186/s12889-018-58796. PubMed PMID: 30081857; PubMed Central PMCID: PMCPMC6090745. 
19. Drewes JL, White JR, Dejea CM, Fathi P, Iyadorai T, Vadivelu J, et al. High-resolution bacterial 16S rRNA gene profile meta-analysis and biofilm status reveal common colorectal cancer consortia. NPJ Biofilms Microbiomes. 2017;3:34. doi: 10.1038/s41522-017-0040-3. PubMed PMID: 29214046; PubMed Central PMCID: PMCPMC5707393.

20. Allen J, Hao S, Sears CL, Timp W. Epigenetic changes induced by Bacteroides fragilis toxin (BFT). Infection and immunity. 2019. doi: 10.1128/IAI.00447-18. PubMed PMID: 30885929.

21. Wang T, Cai G, Qiu Y, Fei N, Zhang M, Pang X, et al. Structural segregation of gut microbiota between colorectal cancer patients and healthy volunteers. ISME J. 2012;6(2):320-9. doi: 10.1038/ismej.2011.109. PubMed PMID: 21850056; PubMed Central PMCID: PMCPMC3260502.

22. Farhana L, Antaki F, Murshed F, Mahmud H, Judd SL, Nangia-Makker P, et al. Gut microbiome profiling and colorectal cancer in African Americans and Caucasian Americans. World J Gastrointest Pathophysiol. 2018;9(2):47-58. doi: 10.4291/wjgp.v9.i2.47. PubMed PMID: 30283710; PubMed Central PMCID: PMCPMC6163128.

23. Shin NR, Whon TW, Bae JW. Proteobacteria: microbial signature of dysbiosis in gut microbiota. Trends Biotechnol. 2015;33(9):496-503. doi: 10.1016/j.tibtech.2015.06.011. PubMed PMID: 26210164.

24. Hawkesworth S, Moore SE, Fulford AJ, Barclay GR, Darboe AA, Mark H, et al. Evidence for metabolic endotoxemia in obese and diabetic Gambian women. Nutr Diabetes. 2013;3:e83. doi: 10.1038/nutd.2013.24. PubMed PMID: 23978817; PubMed Central PMCID: PMCPMC3759130. 
25. Cani PD, Osto M, Geurts L, Everard A. Involvement of gut microbiota in the development of low-grade inflammation and type 2 diabetes associated with obesity. Gut Microbes. 2012;3(4):279-88. doi: 10.4161/gmic.19625. PubMed PMID: 22572877; PubMed Central PMCID: PMCPMC3463487.

26. Wigg AJ, Roberts-Thomson IC, Dymock RB, McCarthy PJ, Grose RH, Cummins AG. The role of small intestinal bacterial overgrowth, intestinal permeability, endotoxaemia, and tumour necrosis factor alpha in the pathogenesis of non-alcoholic steatohepatitis. Gut. 2001;48(2):206-11. PubMed PMID: 11156641; PubMed Central PMCID: PMCPMC1728215.

27. Pussinen PJ, Havulinna AS, Lehto M, Sundvall J, Salomaa V. Endotoxemia Is Associated With an Increased Risk of Incident Diabetes. Diabetes care. 2011;34(2):392-7. doi: 10.2337/dc101676. 


\section{Supporting information captions}

Supplementary table 1: The Microbial DNA qPCR Array Intestinal Infection 2 kit (Qiagen, Hilden, Germany) 


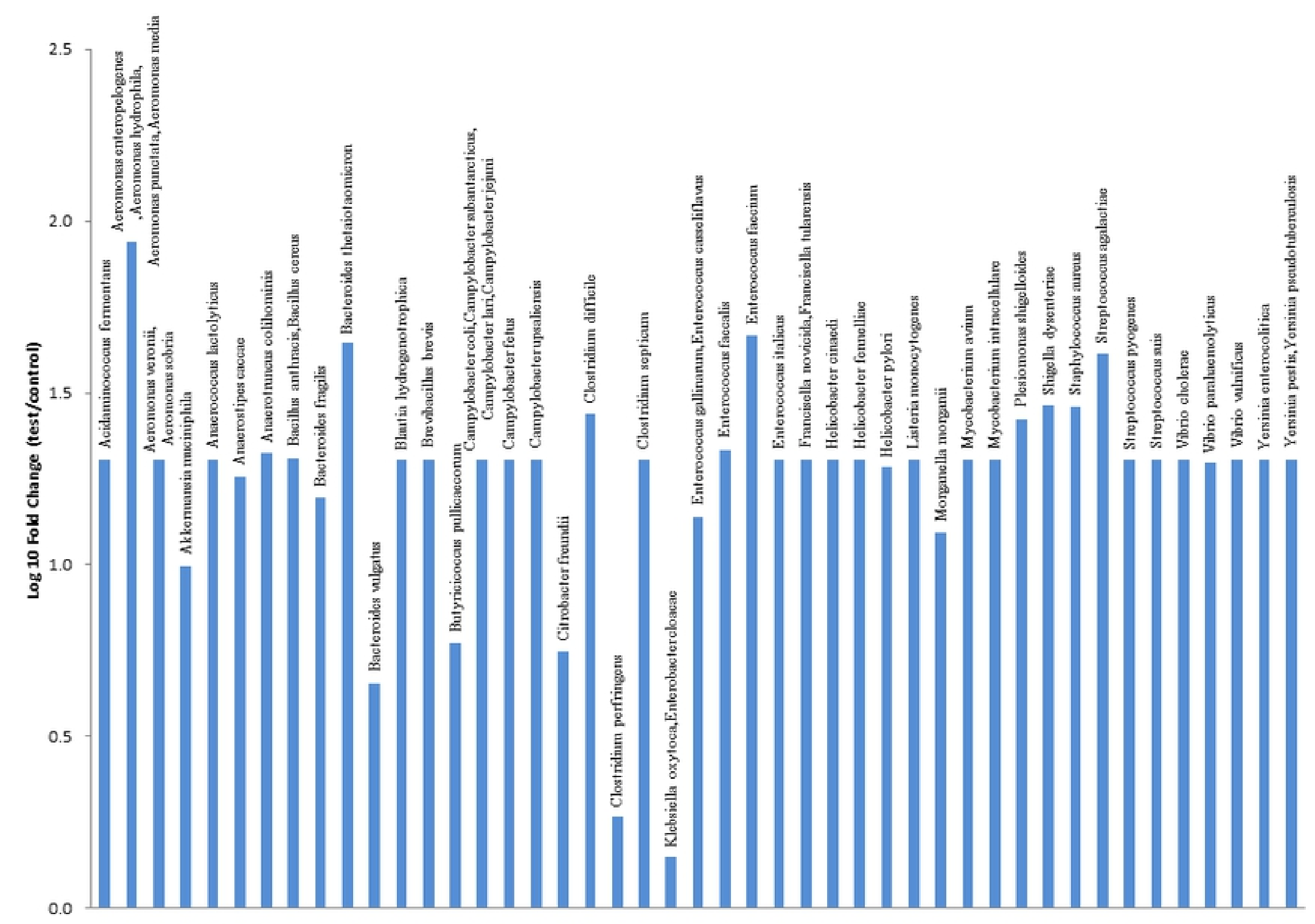

Figure 2 
